\title{
Pharmacotherapy for Borderline Personality Disorder: an Update of Published, Unpublished and Ongoing Studies
}

\author{
Jutta Stoffers-Winterling ${ }^{1} \cdot$ Ole Jakob Storeb $\varnothing^{2,3} \cdot$ Klaus Lieb $^{1}$
}

Published online: 5 June 2020

(C) The Author(s) 2020

\begin{abstract}
Purpose of the Review We aim to identify the most recent evidence of randomised controlled trials evaluating continued drug treatments in people with a diagnosis of BPD, review the most recent findings, highlight trends in terms of currently ongoing studies and comment on the overall body of evidence.

Recent Findings We identified seven new RCTs, plus newly available data for an older RCT. Only three of these RCTs have been published in full text, while we found study data posted at trial registry platforms for the others.

Summary The new findings do not support fluoxetine as a treatment option for suicide and self-harm prevention. A large effectiveness study did not detect beneficial effects of lamotrigine in routine care. The prevalent use of medications in BPD is still not reflected or supported by the current evidence. More research is needed regarding the most commonly used substances and substance classes, i.e. SSRIs, and quetiapine, but also with respect to people presenting with distinct comorbid conditions.
\end{abstract}

Keywords Borderline personality disorder $\cdot$ Drug treatment $\cdot$ Review $\cdot$ Antidepressants $\cdot$ Antipsychotics $\cdot$ Anticonvulsants

\section{Introduction}

Considering the numbers of randomised controlled trials (RCTs) testing drug treatments for borderline personality disorder (BPD), it becomes clear that throughout the last 5 years, this topic seems to be paid less attention to (Fig. 1). Even though some late 2019 papers may not yet have been identified in our March 2020 search, the trend is evident: drug treatments are paid less attention to in current research. This observation is in line with recommendations of the major clinical guidelines for BPD, which concordantly recommend psychotherapy as the first-line treatment $[1$, 2]. It seems that the therapeutic pessimism, which once prevailed

This article is part of the Topical Collection on Personality Disorders

Jutta Stoffers-Winterling

jutta.winterling@gmail.com

1 Department of Psychiatry and Psychotherapy, University Medical Center of the Johannes Gutenberg University Mainz, Untere Zahlbacher Straße 8, D-55131 Mainz, Germany

2 Center for Evidence-Based Psychiatry, Psychiatric Research Unit, Slagelse, Region Zealand Psychiatry, Denmark

3 Department of Psychology, Faculty of Health Sciences, University of Southern Denmark, Odense, Denmark for psychotherapeutic interventions in BPD, now holds for drug treatment. This is not surprising giving the rapidly accumulating evidence of psychotherapy evaluation studies in BPD, and encouraging findings: whereas the previous corresponding Cochrane review included 28 randomised controlled trials in 2012 [3], the 2020 update identified 75 such studies [4]. New systematic reviews and meta-analyses support beneficial effects of psychotherapeutic interventions on relevant clinical outcomes such as psychosocial functioning, BPD severity or self-harming and suicidal behaviour $[5,6]$.

Nevertheless, most people with a diagnosis of BPD receive medications of a large variety of substance classes and oftentimes many drugs concurrently $[7,8]$. Reasons are the absence of psychotherapy [9], comorbid conditions requiring medication, or the addition of new substances in times of crisis, which, in the absence of regular medication reviews, simply accumulate over time.

Facing this clinical situation, it seems worthwhile to keep track of research in the field. New substances and even substance classes have been suggested, especially if specific comorbidities like depressive disorders are present. Building on our 2015 review [10], we aim to follow up recent developments in the field of pharmacotherapy for BPD, and critically discuss and integrate the new studies in the existing body of evidence. We will also highlight ongoing research. 
Fig. 1 Randomised and quasirandomised controlled trials of drug treatments in people with personality disorders.

Publications per year, 1980 to current; CENTRAL search 29 March 2020 (MeSH descriptor [borderline personality disorder] AND qualifier "drug therapy")

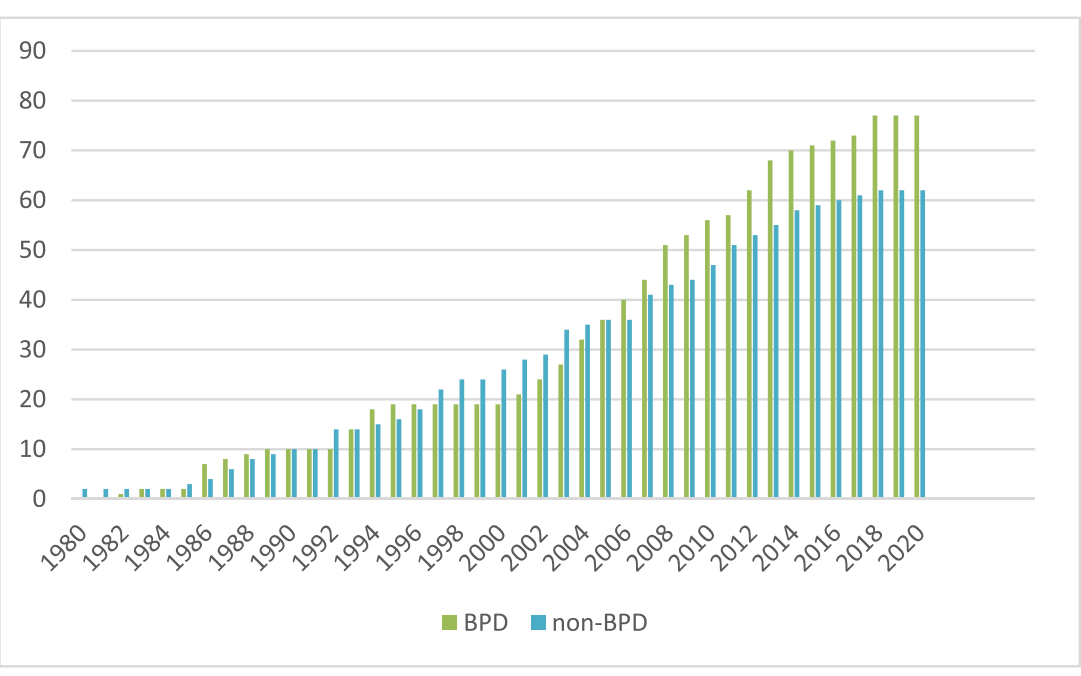

\section{Methods}

We focused on randomised controlled trials (RCTs) of drug treatments for people with borderline personality disorder that reported clinical outcomes. We did not consider studies that concentrated on neurobiological outcomes, or which tested the immediate effects of a once-administered substance. We searched the CENTRAL database of the Cochrane Library in March 2020 for relevant publications from 2015 to present. We also traced back references of study trial register records that we had cited as ongoing studies in our 2015 review [10] in order to identify corresponding full publications. Last, we searched trial registers, i.e. the EudraCT register using the term "borderline personality disorder", the ISRCTN registry (studies with inclusion criterion "borderline personality disorder"), ClinicalTrials.com "Borderline Personality Disorder AND interventional studies"), and the WHO meta-register ICTRP ("borderline personality disorder").

If study results were posted on trial registries without statistical analysis, we statistically compared treatment groups by calculating either standardised mean differences (SMDs) for continuous outcomes or Risk Ratios (RRs) for dichotomous outcomes from post-treatment values, data permitting.

\section{Results of the Search}

Focusing on publication dates 2015 to current (March 2020), we identified five full-text publications $[11 \bullet, 12 \bullet, 13 \bullet, 14 \bullet$, $15 \cdot \bullet$ reporting on four different RCTs with clinical outcomes. One of these records [14 $]$ reported additional data of the Black et al. 2014 RCT [16॰], which had already been included in the 2015 review [10]. Two of these records referred to the same study $[12 \bullet \bullet, 15 \bullet \cdot$. Searching trial platforms, we identified new study data for five RCTs. One referred also to the Black 2014 RCT [17•], whereas four related to RCTs that have not been published elsewhere $[18 \bullet, 19 \bullet, 20 \bullet, 21 \bullet]$. Table 1 gives an overview of the main characteristics of these studies.

\section{Antidepressants}

\section{Recently Published RCT Data}

We did not identify any new full publication of a controlled study or RCTs testing antidepressants in people affected by BPD. Following up the studies which we had listed as ongoing in our previous review [10], we found data posted on ClinicalTrials.gov for two antidepressant RCTs $[18 \bullet$, 19•]. Both these trials investigated fluoxetine. From our calculation of available data, we did not find significantly different rates of serious adverse events (AE) if fluoxetine was compared with placebo [19•]. In contrast, from the RCT, which compared fluoxetine to Dialectical Behavioural Therapy [18•], we observed a significantly higher rate of suicide attempts in the medication-only group (Table 1).

\section{Unpublished RCTs}

When following up the references that we had identified as ongoing in our 2015 review, we found that three were still unpublished: One RCT of escitalopram had never been started [22], whereas another RCT of escitalopram was terminated because of difficulties in recruiting participants [23]. A third RCT testing the MAO-I selegiline against placebo was completed, but though our best efforts, we were not able to find any corresponding publication [24]. The latter was the only industry-sponsored trial among the listed unpublished studies.

\section{Ongoing RCTs}

We did not identify any ongoing RCT evaluating the effects of continued antidepressant treatment. 


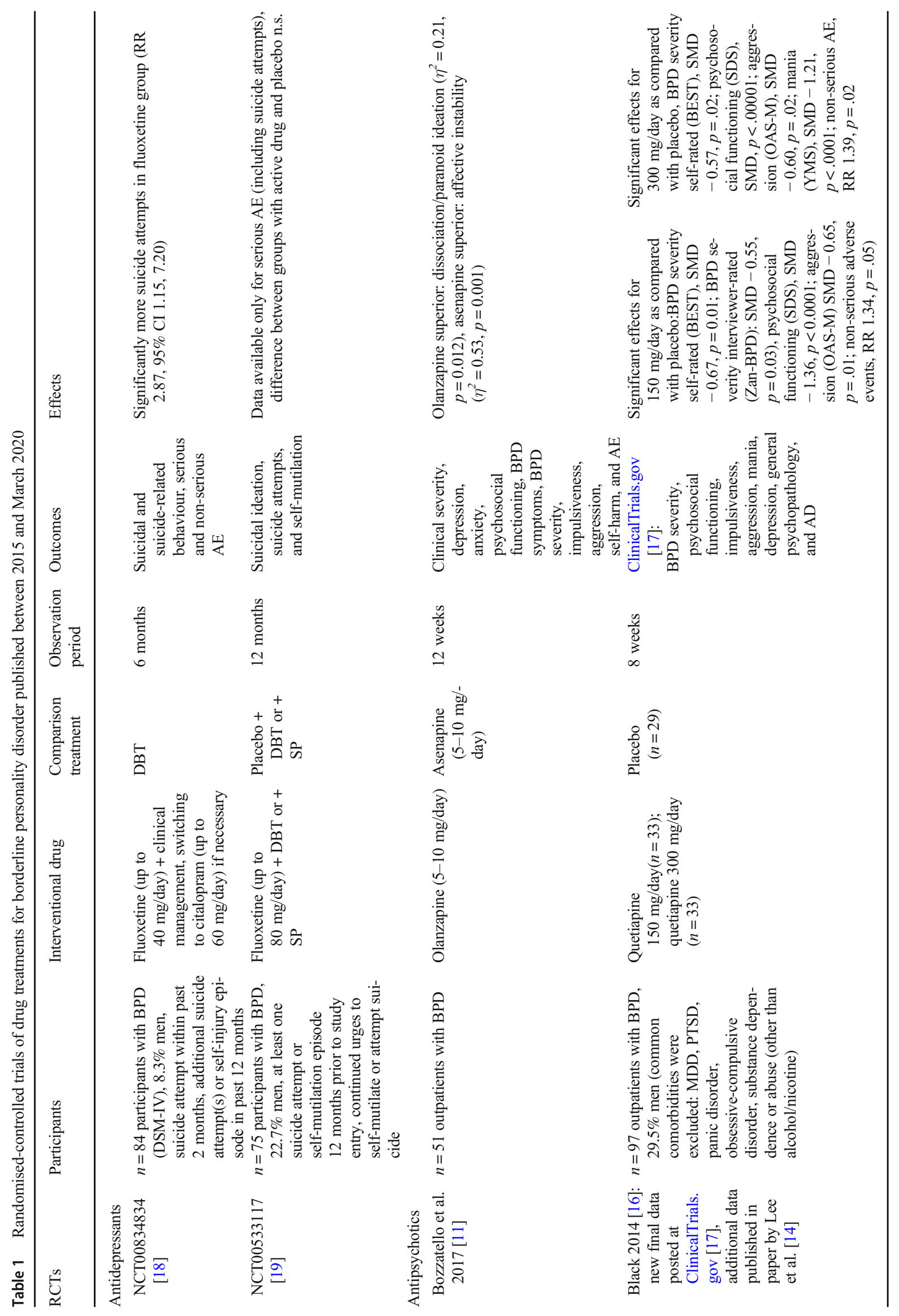




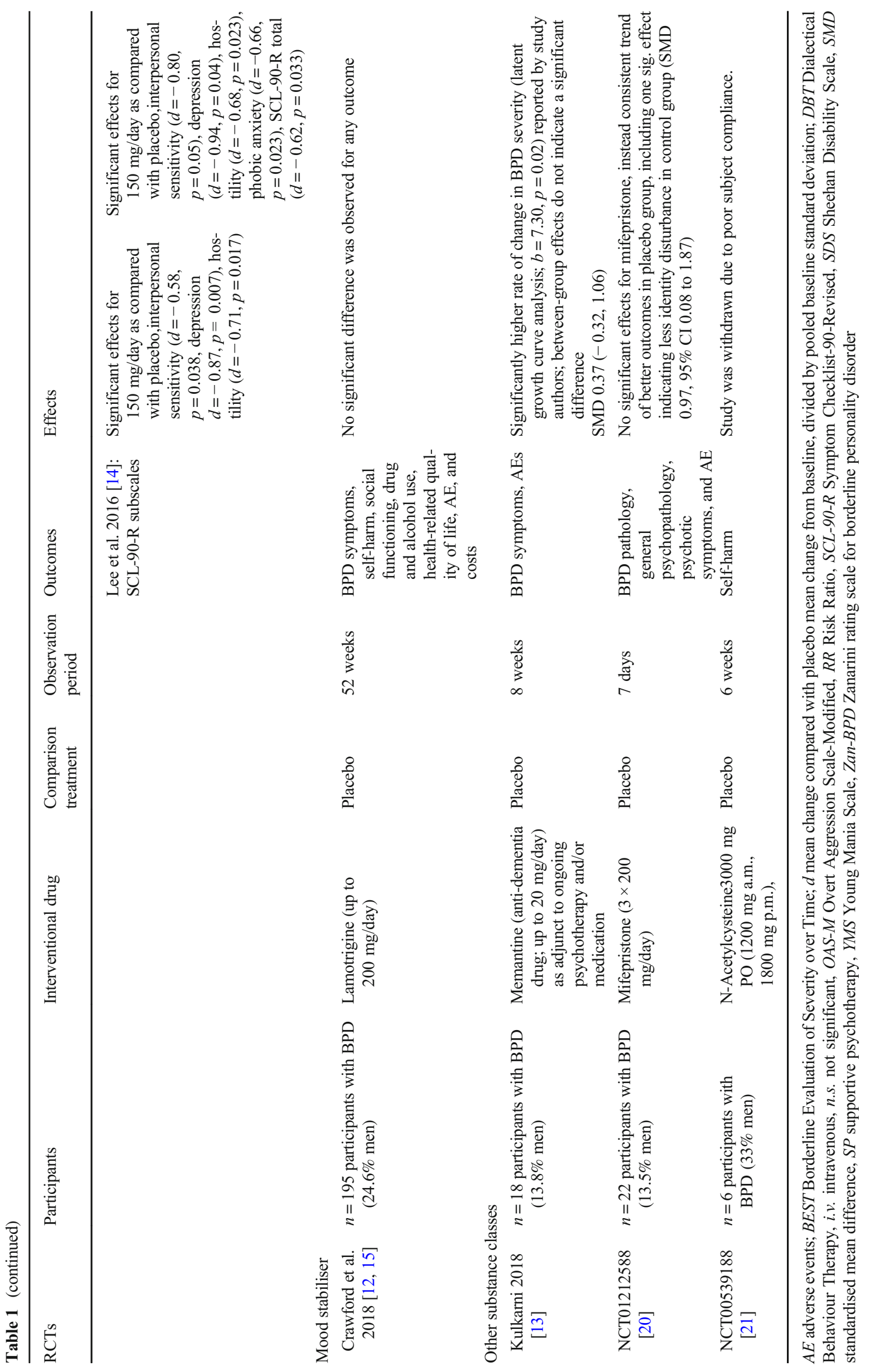




\section{Critical Judgement of Current Evidence}

Given the high rates of antidepressant use in BPD, the lack of relating evidence is startling. The most recent placebocontrolled RCT testing an antidepressant is still the one of Simpson and colleagues, published in 2004 [25]. Large cohort studies concurrently show that antidepressants are the medication class which is most often prescribed, with rates ranging between 70 and $80 \%$ [8, 26-28]. Of course, depressive disorders are highly prevalent in BPD, and if a manifest depressive disorder is present, it may require medication. Yet, another study which compared the medication rates of those inpatients with BPD who had a comorbid depressive disorder to the medication rates of those who had not did not find a difference in the type and number of medications [29]. Paton and colleagues report antidepressant use in $94 \%$ of patients with an emotionally unstable personality disorder (EuPD) who also had comorbid depression, but also in $75 \%$ of people who had EuPD as their sole psychiatric diagnosis [27]. Obviously, comorbidity does not account for the high use of medication in BPD. Despite the complete lack of reliable, supporting evidence, be it from meta-analytically accumulated evidence or at least single RCTs, antidepressants are still prescribed "by default", it seems [30].

\section{Antipsychotics}

\section{Recently Published RCT Data}

We were able to identify one new RCT which compared the effects of the two second-generation antipsychotics (SGAs) olanzapine and asenapine [11•], and two records [14•,17•] adding new data to a yet published placebo-controlled RCT of quetiapine [16•] (s. Table 1). Bozzatello and colleagues compared olanzapine and asenapine head to head and observed very limited differences: olanzapine was superior regarding dissociation, asenapine regarding affective instability. Unfortunately, the trial did not include a placebo control group, so this trial compared two substances of uncertain efficacy. To date, we cannot conclude from the evidence that olanzapine was a helpful and safe treatment option [10,31], and asenapine has never been subject to a placebo-controlled RCT in BPD so far. Even if asenapine had been found to be convincingly superior to olanzapine, it would still be uncertain if patients would profit at all. Given the limited therapeutic effects on the one hand and the well-documented adverse effects of both substances that were also observed within this trial (asenapine: oral hypoesthesia, anxiety/restlessness, akathisia; olanzapine: weight gain, somnolence, fatigue) on the other, neither of the two options seems to be a treatment option.

New data have become available for the only one existing placebo-controlled RCT of quetiapine [16•]. The authors posted final raw data at the ClinicalTrails.gov website [17•], which allow for calculating between-group effect sizes at the time of post-treatment. Using these data, we observed moderate to large, statistically significant effects for both doses of quetiapine ( $150 \mathrm{mg} /$ day and $300 \mathrm{mg} /$ day) regarding BPD severity, psychosocial impairment and aggression, and an additional effect for the higher dose regarding manic symptoms (s Table 1). Comparing the two active groups, we found no superiority of any group. Notably, there was no clear doseeffect relationship for clinical outcomes, i.e. beneficial effects of treatment, but a higher proportion of participants experiencing adverse events in the group with the higher dose. Both doses were associated with significantly heightened rates of adverse events, the most prevalent ones being sedation, mouth dryness, change in appetite and headache. These newer data support the previously reported findings published in the 2014 paper [16•].

\section{Ongoing Studies}

Two placebo-controlled RCTs are currently underway to evaluate the effects of brexpiprazole [32,33], an SGA which has been described as a serotonin-dopamine activity modulator (SDAM). It has been approved for the treatment of schizophrenia by the Food and Drug Administration (FDA) and by the European Medicines Agency (EMA), but it is additionally approved as an adjunctive to antidepressants in major depressive disorder treatment in the USA. Another placebocontrolled RCT is underway in the UK where inpatients with BPD who did not experience an adequate clinical response to antipsychotic medications will receive clozapine [34]. Last, we are aware of another placebo-controlled RCT of aripiprazole for people with a diagnosis of BPD and auditory verbal hallucinations, but without schizophrenia or any psychotic disorder [35]. Notably, this will be one of the first RCTs ever investigating the beneficial and harmful effects of pharmaceutical treatments in adolescents with BPD (minimum age, 15 years; maximum age, 25 years). For an overview of ongoing studies, see Table 2.

\section{Critical Judgement of Current Evidence}

International studies concordantly report antipsychotics being prevalently prescribed to people with a diagnosis of BPD, with rates ranging between 70 and $79 \%$ of inpatients $[8,29$, 36 and 35 and $60 \%$ of outpatients [26-28]. Given the current evidence, it takes wonder why quetiapine plays such an outstanding role within BPD treatment. Among antipsychotic agents, quetiapine is the one which is most often described to people with a diagnosis of BPD, and, above all, it is also the one single substance most often described among all substance classes: actually, every fifth to almost third inpatient would receive quetiapine $[8,29,36]$. To date, only one single 


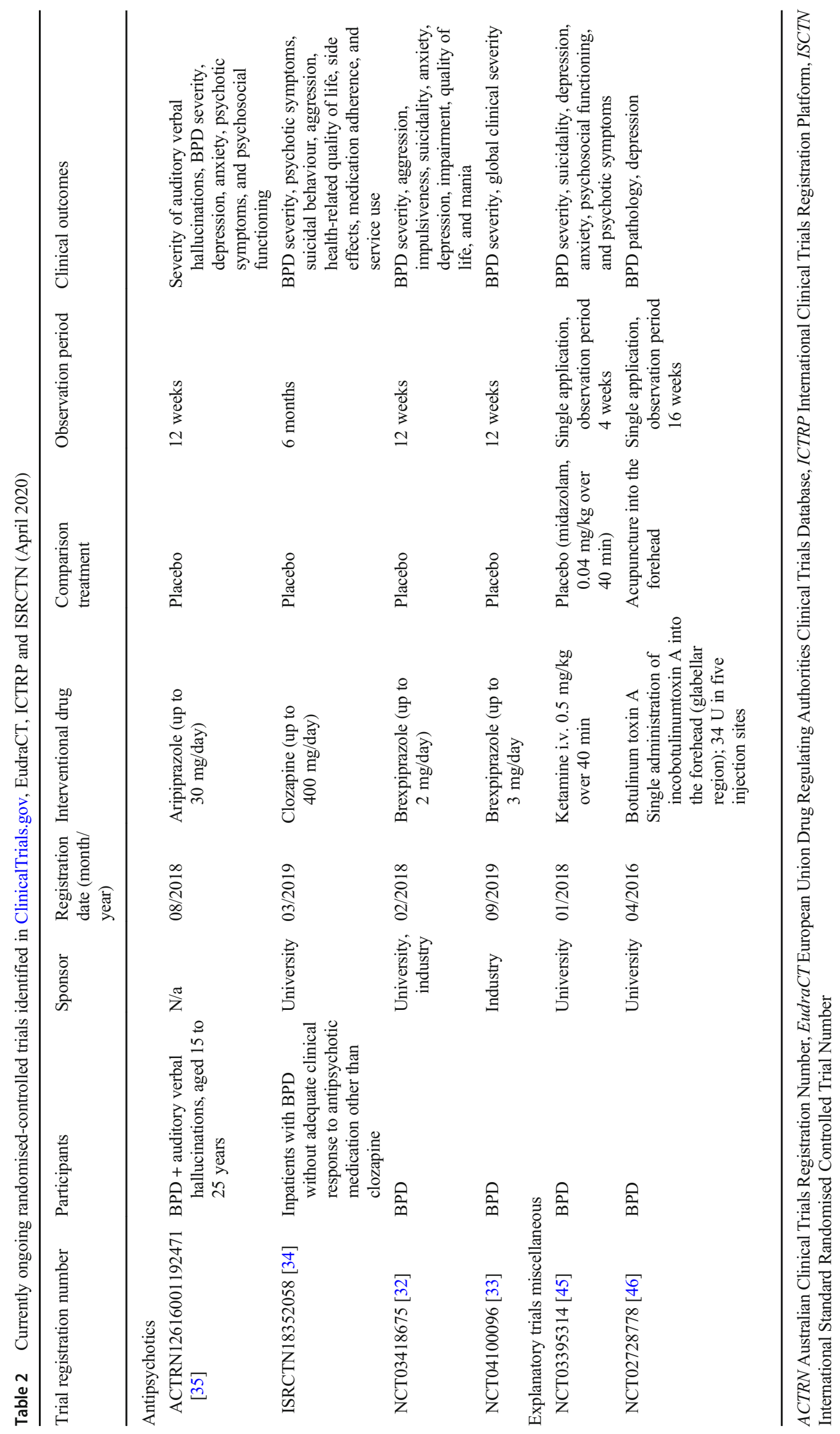


$\operatorname{RCT}[14 \bullet, 16 \bullet, 17 \bullet]$ has ever been published that evaluated the effects of this substance in people with BPD. At the same time, we are aware of a number of RCTs involving quetiapine that have been completed, but results have not been published $[37,38]$. Another RCT involving the antipsychotic risperidone has been registered and was recruiting, but we do not know if this trial has ever been completed, and if so, what the results were [39]. In our previous Cochrane review [31], we had included eight placebo-controlled RCTs of secondgeneration antipsychotics (SGAs; aripiprazole, olanzapine, ziprasidone), but did not observe robust evidence of clinically relevant effects for any of the substances. Instead, we found significant adverse effects for olanzapine, including metabolic changes and weight gain, and indications of more selfharming behaviour under olanzapine. Since our Cochrane review in 2010 [31, 40], only one placebo-controlled RCT of any SGA has been published [16•], indicating some effects of quetiapine regarding psychosocial functioning, BPD severity, and aggression, but also significant adverse events.

\section{Mood Stabilisers}

\section{Recently Published RCT Data}

A large RCT study has been conducted by Crawford and colleagues $[12 \bullet \bullet, 15 \bullet \bullet$. They compared the effects of 12 months of lamotrigine treatment to placebo and observed not one significant difference between the groups. Including a large sample of 276 participants, the study had enough power to detect clinically relevant differences, had there been any. Other strengths of this study are the long observation period (whereas most pharmacotherapy trials last for 12 weeks only on average [31]), and the broad inclusion criteria (only comorbid bipolar and psychotic disorders were excluded). The authors conclude that lamotrigine is neither a clinically nor a cost-effective treatment option for BPD.

\section{Ongoing Studies}

Notably, we were not able to identify any ongoing study of mood stabilisers for the treatment of BPD.

\section{Critical Judgement of Current Evidence}

Previously, mood stabilisers had been reputed a possible alternative role to antipsychotic agents [41, 42]. In our previous Cochrane review from 2010, we also observed some effects for the mood stabilisers lamotrigine, topiramate, and valproic acid [31, 40], and the meantime publication of another, small RCT [43] did not change the results substantially [10]. However, the underlying studies were small, with samples ranging between 15 and 56 participants, included short observation periods and partly applied strict exclusion criteria.
Therefore, the new findings of lamotrigine treatment in routine care shed a completely new, more critical light on the use of mood stabilisers. Weighing uncertain beneficial against possible adverse (i.a. teratogenic) effects, mood stabilisers are not an expedient treatment option in a population of patients, most of which are women in childbearing age.

\section{Explanatory Trials}

\section{Recently Published RCT Data}

Memantine, a substance anti-dementia drug that targets the glutamatergic system, has been tested against placebo [13•]. Many people with BPD have chronically been exposed to stress due to maltreatment during childhood. Chronic stress can induce changes in glutamate release and glutamate receptor functioning, resulting in overactivity and excitotoxicity. By moderating glutamatergic neurotransmission, the NMDA antagonist memantine may reduce BPD severity, and selfharming and impulsive behaviour specifically, which all have been found to be correlated to glutamate concentrations [44]. The study authors report a significant higher rate of change of BPD severity by memantine in a latent growth curve analysis. Calculating the SMD from post-treatment group data, we could not replicate a significant effect (SMD $0.37(-0.32$, $1.06), p=.30)$. Instead, the final BPD severity score was higher in the memantine group.

We identified another placebo-controlled RCT without full-text publication, but with outcome data posted at the ClinicalTrials.gov registry [20•]. In this study, the effects of mifepristone, a glucocorticoid receptor antagonist that is also used as abortifacient, are tested. Mifepristone is supposed to alter a putative hyper-responsiveness of the hypothalamicpituitary-adrenal (HPA) axis. Calculating between-group effects at post-treatment from the posted raw data, we did not find any statistically significant effect favouring mifepristone, but a consistent trend throughout all reported measures of better results in the placebo group. These findings speak against a possible role for mifepristone in BPD treatment.

Last, we found a reference relating to a small placebocontrolled study of $N$-Acetylcysteine (ACC) as an adjunct to an ongoing Dialectical Behavior Therapy (DBT) [21•]. However, the posted study results refer to six participants, of which two completed the 6-week study (one in each group). The authors state that the study was terminated due to poor subject compliance.

\section{Ongoing Studies}

We are aware of an ongoing RCT testing the effects of ketamine against a control treatment with the relaxant midazolam [45]. Ketamine has been observed to rapidly reduce suicidality and improve mood in patients with MDD. This study aims to 
assess if these effects can also be found in people with a diagnosis of BPD.

Last, the effects of applicating botulinum toxin A, a relaxant that is injected into the forehead, are studied in an ongoing RCT [46]. Application of botulinum toxin A results in a paralysis of facial muscles that are involved in the expression of negative emotions. Thus, the afferent feedback is interrupted, which putatively alleviates depressive symptoms. Participants allocated to the control condition will receive acupuncture sessions. Outcome assessment includes depression as well as BPD pathology.

\section{Conclusions}

As in our 2015 review, we must conclude that the evidence of drug treatment effects has not accumulated substantially, a finding which is almost identical to our conclusion 5 years ago. However, the new findings of no beneficial effects by fluoxetine in the prevention of suicidal behaviour in people with BPD (but inferiority to psychotherapy treatment instead) merit closer attention. SSRIs being among the most often used substances in BPD, regardless of co-existing comorbidities, it is remarkable that only three placebo-controlled RCTs of SSRIs in BPD exist, involving, altogether, data of 85 participants $[25,47,48]$.

Another remarkable new finding is the failure to detect beneficial effects by lamotrigine treatment in a real-world effectiveness study $[12 \bullet, 15 \bullet \cdot$. This large-scale pragmatic RCTs yield conclusive results that are applicable routine care, suggesting that lamotrigine is not a clinically effective treatment for people with BPD.

The drug industry has retracted from initiating largescale trials in recent years. Eli Lilly had sponsored two large-scale RCTs of olanzapine [49, 50], which included more than 300 participants each, but did not result in drug approval for use in BPD. After that, there were no major industry-funded research efforts. Medication use being still off-label in BPD, drugs are nevertheless extensively prescribed to people with BPD. The lack of conclusive supporting evidence seems not to hinder the use of medications in routine care. For example, quetiapine has been found to be the single substance most often described to people with BPD at a time when not a single placebocontrolled RCT had been available that would have evaluated the effects of quetiapine in BPD at all [8]. Furthermore, comorbidities like affective, anxiety or traumatic disorders are common in BPD [51], and these conditions may warrant medication use, which is in line with recommendations of the major BPD guidelines $[1,2]$. However, data from clinical practice indicate that drug use is not related to comorbidity [27, 29].
We are facing a manifest gap between evidence and practice. In order to enable people affected by BPD as well as clinicians to make informed decisions, more RCTs are needed. On the one hand, these should replicate previous research where positive findings were observed in single, small studies $[31,40]$. On the other hand, underresearched areas need to be approached: Medication use (especially SSRI) is oftentimes justified by the prevalence of comorbid disorders, especially depression, but there is a clear lack of studies that focused on participants with distinct comorbidities. In terms of interventions, we need more RCTs evaluating the highly used drugs like SSRIs, and quetiapine. Fortunately, there are first new efforts from the industry to evaluate new substances in explanatory trials $[32,33]$. As long as there is no definite conclusion about any substance being effective, trials should include a placebo comparison group. Outcome assessment should include BPD-specific pathology, but also pathology related to comorbid conditions if comorbid samples are in focus. Psychosocial functioning has been found to be crucial for judging mid- and long-term effects [52] and should also be assessed. Adverse events should regularly be monitored in a standardised way. Last, observation periods should last sufficiently long to reflect clinical practice and to allow for drawing conclusions about beneficial effects. Our working group is currently working on an update of the 2010 Cochrane review [53]. Based on comprehensive searches, we intend to review and statistically integrate the available evidence. In this Cochrane review, we will also include a quality assessment of the included trials which we have not done in this review.

Funding Information Open Access funding provided by Projekt DEAL.

\section{Compliance with Ethical Standards}

Conflict of Interest Jutta Stoffers-Winterling, Ole Jakob Storebø and Klaus Lieb each declare no potential conflicts of interest.

Human and Animal Rights and Informed Consent This article does not contain any studies with human or animal subjects performed by any of the authors.

Open Access This article is licensed under a Creative Commons Attribution 4.0 International License, which permits use, sharing, adaptation, distribution and reproduction in any medium or format, as long as you give appropriate credit to the original author(s) and the source, provide a link to the Creative Commons licence, and indicate if changes were made. The images or other third party material in this article are included in the article's Creative Commons licence, unless indicated otherwise in a credit line to the material. If material is not included in the article's Creative Commons licence and your intended use is not permitted by statutory regulation or exceeds the permitted use, you will need to obtain permission directly from the copyright holder. To view a copy of this licence, visit http://creativecommons.org/licenses/by/4.0/. 


\section{References}

Papers of particular interest, published recently, have been highlighted as:

- Of importance

•- Of major importance

1. NHMRC. Clinical practice guideline for the management of borderline personality disorder [Internet]. Canberra: National Health and Medical Research Council; 2013 [cited 2016 Dec 1]. Available from: http://www.nhmrc.gov.au/guidelines/publications/ $\mathrm{mh} 25$

2. NICE National Institute for Health and Care Excellence. 2018 Surveillance of personality disorders (NICE guidelines CG77 and CG78). Surveillance report. Published 19 July 2018. Available from https://www.nice.org.uk/guidance/cg78/resources/2018surveillance-of-personality-disorders-nice-guidelines-cg77-andcg78-4906490080/chapter/Surveillance-decision?tab=evidence. 2018.

3. Stoffers JM, Vollm BA, Rucker G, Timmer A, Huband N, Lieb K. Psychological therapies for people with borderline personality disorder. Cochrane Database Syst Rev [Internet]. 2012; Available from: ://WOS:000308220400007.

4. Storebø OJ, Stoffers-Winterling JM, Völlm BA, et al. Psychological therapies for people with borderline personality disorder. Cochrane Database Syst Rev. 2020;5(5):CD012955. https://doi.org/10.1002/ 14651858.CD012955.pub2.

5. Oud M, Arntz A, Hermens ML, Verhoef R, Kendall T. Specialized psychotherapies for adults with borderline personality disorder: a systematic review and meta-analysis. Aust N Z J Psychiatry. 2018;4867418791257.

6. Cristea IA, Gentili C, Cotet CD, Palomba D, Barbui C, Cuijpers P. Efficacy of psychotherapies for borderline personality disorder: a systematic review and meta-analysis. JAMA Psychiatry. 2017;74: 319-28.

7. Zanarini MC, Frankenburg FR, Reich DB, Conkey LC, Fitzmaurice GM Treatment rates for patients with borderline personality disorder and other personality disorders: a 16-year study. Psychiatr Serv [Internet]. 2015 [cited 2017 Jun 20]; Available from: http://ps.psychiatryonline.org/doi/abs/10.1176/appi.ps. 201400055 ?url_ver $=Z 39.88-2003 \&$ rfr_id=ori\%3 Arid\% 3Acrossref.org\&rfr dat $=\mathrm{cr}$ pub\%3Dpubmed

8. Bridler R, Haberle A, Muller ST, Cattapan K, Grohmann R, Toto S, et al. Psychopharmacological treatment of 2195 in-patients with borderline personality disorder: a comparison with other psychiatric disorders. Eur Neuropsychopharmacol. 2015;25:763-72.

9. Paris J. Why patients with severe personality disorders are overmedicated. J Clin Psychiatry. 2015;76:e521.

10. Stoffers JM, Lieb K. Pharmacotherapy for borderline personality disorder-current evidence and recent trends. Curr Psychiatry Rep. 2015;17:534.

11. Bozzatello P, Rocca P, Uscinska M, Bellino S. Efficacy and tolerability of asenapine compared with olanzapine in borderline personality disorder: an open-label randomized controlled trial. CNS Drugs. 2017;31:809-19 This RCT compared the two secondgeneration antipsychotics olanzapine and azenapine but found no clear superiority of any of the substances. Total efficacy of both agents in people with BPD unclear.

12.• Crawford M, Sanatinia R, Barrett B, Cunningham G, Dale O, Ganguli $\mathrm{P}$, et al. The clinical effectiveness and cost-effectiveness of lamotrigine in borderline personality disorder: a randomized placebo-controlled trial. Am J Psychiatry. 2018;175:756-64 A large-scale, pragmatic RCT. No effects were detected in this effectiveness trial. The long observation period of one year and the broad eligibility criteria for participants grant the findings applicable to routine care.

13. Kulkarni J, Thomas N, Hudaib A-R, Gavrilidis E, Grigg J, Tan R, et al. Effect of the glutamate NMDA receptor antagonist Memantine as adjunctive treatment in borderline personality disorder: an exploratory, randomised, double-blind, placebo-controlled trial. CNS Drugs. 2018;32:179-87 Small experiential RCT testing an anti-dementia drug. Authors report higher rate of change of BPD severity by memantine.

14. Lee SS, Allen J, Black DW, Zanarini MC, Schulz SC. Quetiapine's effect on the SCL-90-R domains in patients with borderline personality disorder. Ann Clin Psychiatry Off J Am Acad Clin Psychiatry. 2016;28:4-10 Additional data relating to [16].

15.• Crawford MJ, Sanatinia R, Barrett B, Cunningham G, Dale O, Ganguli P, et al. Lamotrigine for people with borderline personality disorder: a RCT. Health Technol Assess Winch Engl. 2018;22:168 Comprehensive study report of [12].

16. Black DW, Zanarini MC, Romine A, Shaw M, Allen J, Schulz SC. Comparison of low and moderate dosages of extended-release quetiapine in borderline personality disorder: a randomized, double-blind, placebo-controlled trial. Am J Psychiatry. 2014;171: 1174-82 First and only RCT of quetiapine in people in BPD. New data of this study have been published in [17] and [14].

17. Seroquel extended release (XR) for the management of borderline personality disorder (BPD) - study results - ClinicalTrials.gov [Internet]. [cited 2020 Apr 7]. Available from: https:// clinicaltrials.gov/ct2/show/results/NCT00880919 Study register entry of [16] with study results.

18. Comparing treatments for self-injury and suicidal behavior in people with borderline personality disorder - full text view ClinicalTrials.gov [Internet]. [cited 2020 Mar 29]. Available from: https://clinicaltrials.gov/ct2/show/NCT00834834 Study register entry with study results. Results have not been published elsewhere.

19. Treating suicidal behavior and self-mutilation in people with borderline personality disorder - study results - ClinicalTrials.gov [Internet]. [cited 2020 Mar 31]. Available from: https:// clinicaltrials.gov/ct2/show/results/NCT00533117 Study register entry with study results. Results have not been published elsewhere.

20. Preliminary trial of the effect of glucocorticoid receptor antagonist on borderline personality disorder (BPD) - Full Text View ClinicalTrials.gov [Internet]. [cited 2020 Apr 3]. Available from: https://clinicaltrials.gov/ct2/show/NCT01212588 Experiential trial of mifepristone. The findings do not support the use of this agent.

21. N-Acetylcysteine in adjunct to DBT for the treatment of selfinjurious behavior in BPD - study results - ClinicalTrials.gov [Internet]. [cited 2020 Apr 7]. Available from: https:// clinicaltrials.gov/ct2/show/results/NCT00539188 Earlyterminated RCT due to recruiting problems, study results reported for a very small number of participants.

22. DBT and escitalopram in borderline personality disorder - tabular view - ClinicalTrials.gov [Internet]. [cited 2020 Mar 31]. Available from: https://linicaltrials.gov/ct2/show/record/NCT00255554

23. NCT01103180, University of C, Temple U, Northwestern U, University of Southern M. Selective serotonin reuptake inhibitors (SSRIs) in borderline personality disorder. 2010; Available from: http://clinicaltrials.gov/show/NCT01103180

24. Clinical research study to evaluate selegiline in the treatment of borderline personality disorder - full text view - ClinicalTrials.gov [Internet]. [cited 2014 Jun 24]. Available from: http://clinicaltrials. gov/show/NCT01912391

25. Simpson E, Yen S, Costello E, Rosen K, Begin A, Pistorello J, et al. Combined dialectical behavior therapy and fluoxetine in the 
treatment of borderline personality disorder. J Clin Psychiatry. 2004;65:379-85.

26. Zanarini MC, Frankenburg FR, Reich DB, Harned AL, Fitzmaurice GM. Rates of psychotropic medication use reported by borderline patients and axis II comparison subjects over 16 years of prospective follow-up. J Clin Psychopharmacol. 2015;35:63-7.

27. Paton C, Crawford MJ, Bhatti SF, Patel MX, Barnes TR. The use of psychotropic medication in patients with emotionally unstable personality disorder under the care of UK mental health services. J Clin Psychiatry. 2015;76:e512-8.

28. Martin-Blanco A, Ancochea A, Soler J, Elices M, Carmona C, Pascual J. Changes over the last 15 years in the psychopharmacological management of persons with borderline personality disorder. Acta Psychiatr Scand. 2017;136:323-31.

29. Riffer F, Farkas M, Streibl L, Kaiser E, Sprung M. Psychopharmacological treatment of patients with borderline personality disorder: comparing data from routine clinical care with recommended guidelines. Int J Psychiatry Clin Pract. 2019;23: $178-88$.

30. Ingenhoven $\mathrm{T}$. Pharmacotherapy for borderline patients: business as usual or by default? J Clin Psychiatry. 2015;76:522-3.

31. Stoffers JM, Völlm BA, Rücker G, Timmer A, Lieb K. Pharmacological interventions for borderline personality disorder. Cochrane Database Syst Rev Online. 2010;6.

32. Brexpiprazole in borderline personality disorder - full text view ClinicalTrials.gov [Internet]. [cited 2020 Apr 3]. Available from: https://clinicaltrials.gov/ct2/show/NCT03418675

33. A trial of brexpiprazole in the treatment of borderline personality disorder - tabular view - ClinicalTrials.gov [Internet]. [cited 2020 Apr 6]. Available from: https://clinicaltrials.gov/ct2/show/record/ NCT04100096

34. ISRCTN - ISRCTN18352058: Clozapine in the treatment of borderline personality disorder. The CALMED study. [Internet]. [cited 2020 Mar 31]. Available from: http://www.isrctn.com/ ISRCTN18352058

35. ACTRN12616001192471. VERBATIM: A randomised controlled trial of aripiprazole for the treatment of auditory verbal hallucinations in borderline personality disorder [Internet]. [cited 2018 Sep 16]. Available from: https://www.anzctr.org.au/Trial/Registration/ TrialReview. aspx?id=371038

36. Paolini E, Mezzetti FAF, Pierri F, Moretti P. Pharmacological treatment of borderline personality disorder: a retrospective observational study at inpatient unit in Italy. Int J Psychiatry Clin Pract. 2017;21:75-9.

37. NCT00254748. Verkes borderline study: the effect of quetiapine on borderline personality disordered patients [Internet]. [cited 2018 Sep 16]. Available from: https://ClinicalTrials.gov/show/ NCT00254748

38. ISRCTN11135486 - Quetiapine versus sertraline as the pharmacological component in a standardised psychopharmacological and psychotherapeutic treatment of borderline personality disorder: a randomised, rater-blinded study [Internet]. [cited 2014 Jun 13]. Available from: http://www.controlled-trials.com/ ISRCTN11135486

39. Low-dose risperidone treatment for subjects suffering from borderline personality disorder - tabular view - ClinicalTrials.gov [Internet]. [cited 2020 Apr 6]. Available from: https://clinicaltrials. gov/ct2/show/record/NCT00633802

40. Lieb K, Völlm B, Rücker G, Timmer A, Stoffers JM. Pharmacotherapy for borderline personality disorder: Cochrane systematic review of randomised trials. Br J Psychiatry J Ment Sci. 2010;196:4-12.

41. Feurino L, Silk KR. State of the art in the pharmacologic treatment of borderline personality disorder. Curr Psychiatry Rep. 2011;13: 69-75.

42. Abraham PF, Calabrese JR. Evidenced-based pharmacologic treatment of borderline personality disorder: a shift from SSRIs to anticonvulsants and atypical antipsychotics? J Affect Disord. 2008;111:21-30.

43. Moen R, Freitag M, Miller M, Lee S, Romine A, Song S, et al. Efficacy of extended-release divalproex combined with "condensed" dialectical behavior therapy for individuals with borderline personality disorder. Ann Clin Psychiatry. 2012;24:255-60.

44. Hoerst M, Weber-Fahr W, Tunc-Skarka N, Ruf M, Bohus M, Schmahl C, et al. Correlation of glutamate levels in the anterior cingulate cortex with self-reported impulsivity in patients with borderline personality disorder and healthy controls. Arch Gen Psychiatry. 2010;67:946-54.

45. Ketamine in borderline personality disorder - tabular view ClinicalTrials.gov [Internet]. [cited 2020 Apr 7]. Available from: https://clinicaltrials.gov/ct2/show/record/NCT03395314

46. Botulinum toxin A for emotional stabilization in borderline personality disorder (BPD) - full text view - ClinicalTrials.gov [Internet]. [cited 2020 Apr 3]. Available from: https://clinicaltrials.gov/ct2/ show/NCT02728778

47. Salzman C, Wolfson AN, Schatzberg A, Looper J, Henke R, Albanese $\mathrm{M}$, et al. Effect of fluoxetine on anger in symptomatic volunteers with borderline personality disorder. J Clin Psychopharmacol. 1995;15:23-9.

48. Rinne T, van den Brink W, Wouter L, van Dyck R. SSRI treatment of borderline personality disorder: a randomized, placebocontrolled clinical trial for female patients with borderline personality disorder. Am J Psychiatry. 2002;159:2048-54.

49. Schulz SC, Zanarini MC, Bateman A, Bohus M, Detke HC, Trzaskoma Q, et al. Olanzapine for the treatment of borderline personality disorder: variable dose 12-week randomised doubleblind placebo-controlled study. Br J Psychiatry. 2008;193:485-92.

50. Zanarini MC, Schulz SC, Detke HC, Tanaka Y, Zhao F, Lin D, et al. A dose comparison of olanzapine for the treatment of borderline personality disorder: a 12-week randomized, double-blind, placebo-controlled study. J Clin Psychiatry. 2011;72:1353-62.

51. Shah R, Zanarini MC. Comorbidity of borderline personality disorder: current status and future directions. Psychiatry Clin North Am. 2018;41:583-93.

52. Zanarini MC, Frankenburg FR, Reich DB, Fitzmaurice G. Attainment and stability of sustained symptomatic remission and recovery among borderline patients and axis II comparison subjects: a 16-year prospective follow-up study. Am J Psychiatry. 2012;169:476-83.

53. Stoffers-Winterling JM, Storebø OJ, Völlm BA, Mattivi JT, Nielsen SS, Kielsholm ML, et al. Pharmacological interventions for people with borderline personality disorder. Cochrane Database Syst Rev [Internet]. 2018 [cited 2018 Aug 20]; Available from: https://doi.org/10.1002/14651858.CD012956/ abstract

Publisher's Note Springer Nature remains neutral with regard to jurisdictional claims in published maps and institutional affiliations. 